Portland State University

PDXScholar

$6-2008$

\title{
How Students use Mathematical Resources in an Electrostatics Context
}

\author{
Dawn C. Meredith \\ University of New Hampshire - Main Campus \\ Karen A. Marrongelle \\ Portland State University
}

Follow this and additional works at: https://pdxscholar.library.pdx.edu/mth_fac

Part of the Mathematics Commons, and the Physics Commons

\section{Let us know how access to this document benefits you.}

\section{Citation Details}

Meredith, Dawn C. and Marrongelle, Karen A., (2008). How students use mathematical resources in an electrostatics context. American Journal of Physics, 76, 570-578.

This Article is brought to you for free and open access. It has been accepted for inclusion in Mathematics and Statistics Faculty Publications and Presentations by an authorized administrator of PDXScholar. Please contact us if we can make this document more accessible: pdxscholar@pdx.edu. 


\title{
How students use mathematical resources in an electrostatics context
}

\author{
Dawn C. Meredith ${ }^{\text {a) }}$ \\ Department of Physics, University of New Hampshire, Durham, New Hampshire 03824 \\ Karen A. Marrongelle ${ }^{\text {b) }}$ \\ Department of Mathematics and Statistics, Portland State University, Portland, Oregon 97207
}

(Received 24 July 2006; accepted 10 January 2008)

\begin{abstract}
We present evidence that although students' mathematical skills in introductory calculus-based physics classes may not be readily applied in physics contexts, these students have strong mathematical resources on which to build effective instruction. Our evidence is based on clinical interviews of problem solving in electrostatics, which are analyzed using the framework of Sherin's symbolic forms. We find that students use notions of "dependence" and "parts-of-a-whole" to successfully guide their work, even in novel situations. We also present evidence that students' naive conceptions of the limit may prevent them from viewing integrals as sums. (C) 2008 American Association of Physics Teachers.
\end{abstract}

[DOI: $10.1119 / 1.2839558]$

\section{INTRODUCTION}

Mathematics is essential for most physics courses. As physics teachers, we would like to assume that our students have the prerequisite mathematical skills necessary for success in our courses, yet too often it seems they do not. Students forget how to appropriately apply mathematical tools or work out the details incorrectly. Perhaps students are still struggling with difficult concepts from their earlier mathematics courses. Addressing mathematics competency creates a tension when we believe we must spend time on a review of mathematics at the expense of physics content

We give evidence, building on the work of other physics educators, that students do have useful mathematical resources on which we can build effective and efficient instruction. We base our work on the resource perspective which argues that students have small-grained resources on which they can build and further their knowledge. ${ }^{1-3}$ These resources are neither right nor wrong in themselves, but can be correctly or incorrectly applied. For example, the resource that "closer means stronger" correctly explains why we sit close to a fire on a cold night, but is incorrectly applied when used to explain the seasons on Earth. ${ }^{1}$

Starting from this resource perspective, we investigate how students know when to integrate in an electrostatic context by examining the questions: What resources cue students to integrate in a given context? Are these resources common among students? Are some resources more useful than others in applying integration to novel physics situations? Are any resources commonly misapplied?

In Secs. II and III we give an overview of the related literature on resource theory, specifically Sherin's theoretical work on mathematical resources. ${ }^{4}$ We then outline in Sec. IV the methodology used to gather and interpret data, and in Sec. V give details of the four electrostatics questions asked of the ten student sample. The core of this paper follows in Sec. VI, in which we present how students use mathematical resources to cue integration in electrostatics and extend our work to a larger student population. We speculate in Sec. VII on the developmental nesting of resources in this context. Section VIII outlines some implications for instruction.

\section{RELATED LITERATURE}

\section{A. The integral and related concepts}

Previous investigations into students' understanding of calculus concepts have shown that students can carry out methods of differentiation and integration, but many lack the conceptual underpinnings necessary to explain procedures, use multiple strategies, and connect concepts, even after explicit instruction. ${ }^{5-8}$ This conclusion is similar to findings of student work in physics: Students can carry out the calculations, but many lack the associated conceptual understanding. ${ }^{9}$

A central concept of an integral is as the limit of a Riemann sum. Orton ${ }^{6}$ interviewed 110 British students ages 16-22 on several integration and limit tasks and classified student errors as structural (fundamental or conceptual), executive (operational and procedural), or arbitrary. One question asked students to calculate the sum of five rectangles that approximated the area under a curve; 97 of the 110 students answered this question correctly. The other 13 students had difficulty simplifying their answer. Based on this result, it was concluded that students do not have significant difficulty evaluating a given Riemann sum.

Students were also asked to consider the sequence of areas obtained by using smaller rectangles to approximate the area under a curve. Students' responses to this task indicated that the majority of students view the limit of the Riemann sum as an approximation, not as an exact answer. This result points to the difficulty that students have coordinating their understanding of an integral as a limit.

Cornu $^{10}$ argues that students' previous experiences with the word "limit" contribute to the way in which they make sense of the concept of limit (both functional and sequential) in mathematics classes. For instance, students may define the word limit as a point that is approached but never reached, a constraint, or a high or low point. Cornu calls these colloquial uses of limit spontaneous conceptions, similar to naive conceptions or misconceptions in physics. ${ }^{11}$ Students often view the limit as a dynamic process or as unattainable and have significant difficulty with the concept of limit because of its underlying mathematical ideas, such as function, infinity, and the real number system. ${ }^{10,12-15}$ 


\section{B. Electrostatics}

Work by Harrington ${ }^{16}$ and Kanim ${ }^{17}$ probes student understanding of electrostatics and circuits. Many student difficulties in this area arise from conceptual difficulties with vector superposition; therefore, we have purposely avoided vector issues in this study.

Kanim ${ }^{17}$ analyzed students' answers when they were asked to calculate the net electric field at a given point due to a bar of charge. After direct instruction, only $40 \%$ of the students knew that integration was necessary for a continuous charge distribution and only $10 \%$ did the calculation correctly. Students also had trouble making sense of charge density, either assuming that each point had the charge of the whole, or that each point had a charge so small that the total effect was small compared to a single point with the same total charge. Some students treated the distributed charge as if it were at a point, which is called the "center of charge" in analogy to center of mass. This approach, like representing a country by its leader, attempts to represent the whole by a part; it is named the "part-for-whole" knowledge resource. ${ }^{18}$ We will refer to this resource by its Greek name "synecdoche" to avoid confusion with the "parts-of-a-whole" resource, defined in the next section.

\section{THEORETICAL FRAMEWORK ON STUDENT RESOURCES}

The resource perspective focuses on content-specific primitive cognitive elements and their complex interactions. ${ }^{4}$ Being content-specific, physics resources can be conceptual, epistemological (related to beliefs about learning), mathematical, or otherwise. Being primitive, these knowledge elements seem self-evident to the user; they are not explained or questioned. diSessa's ${ }^{1}$ work on sense of mechanism was the first work in this vein; in Ref. 1 he outlined the primitive intuitive elements, such as "closer means stronger," that we all use to explain the physical world around us.

For our work, we need to understand mathematical resources to help us analyze students' work as they link intuition with mathematical formalism. For this, we turn to the work of Sherin, ${ }^{4}$ who has outlined cognitive mathematical primitives (called symbolic forms) that allow students to "associate meaning with certain structures in equations." As a simple example, the dependence form is associated with the idea that the quantity of interest depends on a variable $x$ and changes when $x$ changes. The associated structure is given by $[\ldots x \ldots]$ and indicates that $x$ appears somewhere in the equation. Symbolic forms allow students to create, modify or complete, evaluate, and extract implications from equations. Sherin summarizes the use of symbolic forms:

"Successful physics students learn to express a moderately large vocabulary of simple ideas in equations and to read these same ideas out of equations. I call the elements of this vocabulary symbolic forms. Each symbolic form associates a simple conceptual schema with an arrangement of symbols in an equation. Because they possess these symbolic forms, students can take a conceptual understanding of some physics situation and express that understanding in an equation. Further- more, they can look at an equation and understand it as a particular description of a physical system."

Sherin's experimental evidence for symbolic forms came from observing five pairs of sophomore engineering students at the University of California, Berkeley who were solving moderately difficult physics problems. Sherin's strongest evidence that students are not only applying known principles is that one pair of students invented a new equation for the coefficient of friction based on their intuition. His data also show that students display much certainty (and even emotion) when invoking symbolic forms. For example, when two students derive the equation $a=g \mu$ for the acceleration of two shoved blocks of different mass on a rough surface (with coefficient of friction $\mu$ ) one student notes, "Wait a minute. Oh, they both take the same time! [surprised tone]. So, no matter what the mass is, you're gonna get the same, the same acceleration." They are able to see something new at once and with great certainty by recognizing the "no dependence" symbolic form in this equation. We infer that symbolic forms can (at least in some circumstances) be effortlessly invoked and applied by students.

Sherin's data corpus included only problems that can be solved algebraically. Our work is an application of Sherin's work in a calculus context. We show that the same symbolic forms are also useful to students and spontaneously invoked by them in problems requiring calculus. We expected to see the parts-of-a-whole symbolic form as the key idea of integration in electrostatics problems. This symbolic form is associated with the idea that several pieces must be added together to make a whole, as when tiny bits of charge are added up to obtain the total charge.

\section{METHODOLOGY}

In the spring of 2000 we conducted individual interviews with 12 students: Six students from an integrated calculus/ physics course and six from a conventional physics course that had integral calculus as a corequisite. The details of the integrated calculus/physics course have been described elsewhere. ${ }^{19}$

Our initial intent in inviting students from two different types of classes was to look for differences in student performance due to different instruction. However, we realized that the similarities between the two groups of students were worth studying in detail and would give us insight into the resources that all students use to solve calculus-based physics problems. Thus, we choose not to compare the two groups of students in this report.

We recruited students of various abilities; these abilities were judged based on grades from their previous calculus and physics courses. Four students had calculus and physics grades of A; four had calculus and/or physics grades of B; and four had calculus and/or physics grades of less than B. We followed the practice of interviewing a small number of students in order to gain detailed insight into student thinking. ${ }^{4,7}$

We conducted three interviews with each student; each interview was an hour long. The first and the last interviews concentrated on problem solving; the second interview focused on the use of calculus to calculate physical quantities. We will discuss only the second interview.

The students were coached in a think aloud protocol during the first interview to help them understand the level of 
Table I. Problem statements.

\begin{tabular}{ll}
\hline \hline Problem & Description \\
\hline 1 & Constant density: Consider a bar of charge with a charge density of $5 \mu \mathrm{C} / \mathrm{m}$. \\
& If the bar is $0.5 \mathrm{~m}$ long, what is the total charge on the bar? \\
& Nonconstant density: Consider a bar of charge with a charge density of \\
& $(5+7 x) \mu \mathrm{C} / \mathrm{m}$, where $x$ is measured in meters and $x=0$ at the left end of the \\
bar. If the bar is $0.5 \mathrm{~m}$ long, what is the total charge on the bar? \\
Electric field: Consider again the bar of charge with a charge density of \\
$5 \mu \mathrm{C} / \mathrm{m}$ and $0.5 \mathrm{~m}$ length. What is the electric field $3 \mathrm{~m}$ directly to the left of \\
the bar? \\
Capacitor: Consider a parallel plate capacitor on which the charge is changing \\
over time so that the electric field is also changed as $E(t)=8$ sin $(5 t) \mathrm{N} / \mathrm{C}$ in \\
the $y$ direction, where the $y$ direction is vertical. The plates are $3 \mathrm{~cm}$ apart and \\
$40 \mathrm{~cm}$ long. An electron with charge $1.6 \times 10^{-19} \mathrm{C}$ and mass $9.11 \times 10^{-31} \mathrm{~kg}$ \\
enters the region between the plates at time $t=0$ with velocity $v_{0}=2 \times 10^{6} \mathrm{~m} / \mathrm{s}$ \\
in the horizontal direction, at a distance midway between the plates. Does the \\
electron hit the plates before leaving? Assume that (at each instant in time) the \\
electric field is constant within the capacitor plate and 0 outside; that is, ignore \\
fringe effects. Also ignore the gravitational force (Ref. 21$).$
\end{tabular}

detail we wanted to hear as they worked on the problems. All interviews were audiotaped and fully transcribed. Two students were omitted due to incomplete interviews, leaving a total of ten. Students were informed that they would be working on electrostatics problems but were not prompted to use calculus as they solved the problems. Setting up the interview context in this way allowed us to determine if students knew when and how to use integration in an electrostatics context. The interviews occurred at least 1 month after the students were tested on electrostatics while students were studying circuits and magnetism.

Interview transcripts were analyzed in several stages. First we noted whether or not students integrated in each problem. If students did not integrate, we looked for evidence that they failed to see essential connections (for example, between acceleration and electric field) or had conceptual difficulties (for example, they were unclear where the charges resided).

For students who used integration to solve the problems, we examined why they used integration. Because both our text $^{20}$ and the curriculum emphasized integration as a sum, we expected that students would describe integration as a process of summation. We were surprised to find a few students who did not mention sums. This omission led to our third stage of analysis, in which we re-examined these interview transcripts for more detail on how the students described integration. Lastly, we looked for the invention of equations using symbolic forms and for instances where symbolic forms did not help students completely solve problems, or even led them astray.

We were surprised to find that what we classified as the most difficult problem (Problem 4, the capacitor problem), was the problem that most readily cued students to integrate. Students struggled on this problem when they failed to connect electric field to acceleration, but once that connection was made, they were able to recall (not invent) the connection between acceleration and position.

\section{ELECTROSTATICS PROBLEMS AND STUDENT RESPONSES}

In this section we give a brief overview of the interview problems (Table I) and student responses (Table II). We ex- plain the cues for integration in the next section. In the following we use pseudonyms for all of the students.

The constant charge density problem did not require calculus to solve, but served to show how students thought about density. It was important to know if students had some understanding of density before attempting more difficult problems.

All ten students answered this question correctly, though not always immediately and often with initial errors or uncertainty. Several students mentioned the process of multiplication. Georgette's response is typical: "[The charge density is] $5 \mu \mathrm{C} / \mathrm{m}$. And the length is $0.5 \mathrm{~m}$, so if you multiply $j$ [charge density] times $L$ [length] you get $2.5 \mu \mathrm{C}$." Other students did not explicitly mention a formula, as is shown in Edward's answer: "So if it's $5 \mu \mathrm{C} / \mathrm{m}$ and that is only $0.5 \mathrm{~m}$, the total charge would be 2.5 .'

The nonconstant charge density problem required integration for solution. Three of the ten students misinterpreted the spatial dependence in the problem. These students interpreted the spatial dependence as a dependence on the length of the bar and not on the distance of a piece of the bar from

Table II. Summary of students' use of integration cues.

\begin{tabular}{|c|c|c|}
\hline Name & Level & Summary \\
\hline Larry & Low & Never integrated. \\
\hline Bruce & Low & Integrated once using recall. \\
\hline Ivan & Low & Integrated once using dependence. \\
\hline David & Medium & Integrated twice using dependence. \\
\hline Edward & Medium & $\begin{array}{l}\text { Integrated three times using dependence and } \\
\text { recall. }\end{array}$ \\
\hline Alex & High & Integrated three times using all three cues. \\
\hline Kevin & High & $\begin{array}{l}\text { Integrated twice (almost) using dependence } \\
\text { and parts-of-a-whole. }\end{array}$ \\
\hline Isaiah & Medium & $\begin{array}{l}\text { Integrated three times using all three cues; } \\
\text { mentioned limits. }\end{array}$ \\
\hline Esther & High & $\begin{array}{l}\text { Integrated three times using all three cues; } \\
\text { mentioned limits. }\end{array}$ \\
\hline Georgette & High & $\begin{array}{l}\text { Integrated twice using parts-of-a-whole and } \\
\text { dependence; mentioned limits. }\end{array}$ \\
\hline
\end{tabular}




\begin{tabular}{lcccc}
\hline \hline & $\begin{array}{c}\text { Used by how } \\
\text { many students? } \\
(10 \text { max })\end{array}$ & $\begin{array}{c}\text { Used in how } \\
\text { many solutions? } \\
(30 \text { max })\end{array}$ & $\begin{array}{c}\text { Used in which } \\
\text { problems? }\end{array}$ & $\begin{array}{c}\text { Used with other } \\
\text { cues? }\end{array}$ \\
\hline $\begin{array}{l}\text { Recall } \\
\begin{array}{l}\text { Dependence symbolic } \\
\text { form }\end{array}\end{array}$ & 5 & 5 & Capacitor only & No \\
$\begin{array}{l}\text { Parts of a whole } \\
\text { symbolic form }\end{array}$ & 5 & 14 & All three & Yes \\
\hline \hline
\end{tabular}

the origin, relieving them of the need to integrate. The remaining seven students understood the density equation as it was intended, each mentioning in some way that the density varies along the length of the bar. All of them used integration to solve the problem.

As students attempted to solve the electric field problem, we observed a large spectrum of student skills and understandings. Three students struggled with the physics concepts; each introduced ideas from several places (for example, bar magnets and capacitance). They were unable to make much headway even after they were given the formula for the electric field due to a point charge and did not see the need to integrate. Two other students solved this problem using the center of charge method. ${ }^{16,17}$ The remaining five students used integration to solve this problem.

The capacitor problem is nontrivial: The motion is twodimensional, the acceleration is given only indirectly and changes in time, and the position is necessary to solve the problem. The physical situation was unfamiliar to the students, though many made useful analogies to motion in a constant gravitational field. Additionally, the magnitudes of the quantities were confusing to some students: The time the electron is inside the capacitor is beyond their experience. A complete solution would require solving a transcendental equation; none of the students got far enough to see this difficulty.

Four students were unsuccessful, primarily because they did not see that the connection between electric field and acceleration was a necessary part of the solution. The remaining six students realized that integrating the acceleration would yield the velocity and the position, although there was a range of abilities in carrying out that plan.

\section{STUDENT RESOURCES USED IN INTEGRATION PROBLEMS}

At this point we return to our main questions: What resources do students use to help them integrate in physics contexts? Are these resources common among students? Are some resources more useful than others in being able to integrate in nontrivial or novel physics situations? In this section we give some of the details of student answers, organized by the three different cues for integration that we found students used: Recall, the symbolic forms of dependence, and parts-of-a-whole. Failure to integrate is also discussed. These results and the students' approaches to solving the four problems are summarized in Tables II and III.

Within each cue, quotations are organized by question: Nonuniform density, electric field, and capacitor. We have included some long and less than clear comments by the students and we speculate in Sec. VII about what we can learn from students' lack of clarity. We have deleted some of the interviewer's simple prompts. We begin each cue with a description of how the cue is identified, building on Sherin's work for the identification of symbolic forms.

One student, Larry, did not employ integration in his solutions for any of the problems. We are unable to determine if his lack of progress arose from difficulties with physics, calculus, or both. Thus, we present an analysis of the nine remaining students' approaches to the problems.

\section{A. Integration not cued}

Five of the nine students failed to integrate in at least one problem. Three students did not integrate in the nonconstant density problem because they interpreted $x$ to be the total length of the bar and thus misinterpreted the dependence in the equation. In the electric field problem, four students failed to integrate. Ivan and David used the incorrect center of charge method. Neither Georgette nor Bruce integrated in this problem, and we infer this lack was due to their struggle with the physics concepts. Both Georgette and Bruce brought in several related but irrelevant ideas (dipoles, perpendicular and parallel fields, magnetic fields, and capacitance). In the capacitor problem, Larry and Ivan failed to integrate; they could not have been cued to integrate by any method because they did not recall the relation between force and acceleration.

In all cases in which integration was not cued, there was evidence that a misunderstanding of the physics prevented the student from being able to see the need to integrate; however, we cannot conclude that if they had understood the physics, they would have been cued to integrate. There were two cases where clearing up confusion about the physical situation allowed students to solve the problem confidently. In one case, Isaiah correctly calculated the charge in the constant density problem. He was not confident in his answer because he was not sure that the density was a smooth function; he instead focused on the discreteness of the electrons. Once the interviewer suggested that he assume the total charge in each same sized chunk is equal (acknowledging that this assumption is an idealization), he was confident that his answer was correct. The second case appeared in the electric field problem. Kevin was stuck for a long time, not knowing if the charge on the bar is allowed to move. Once he was asked what he would do if the charge were evenly spread over the bar, he knew immediately to integrate, and he proceeded nearly correctly and with confidence.

We conclude that an understanding of the physical situation is necessary, but not sufficient for students to use their mathematical resources. This idea is not new, but bears repeating. 


\section{B. Recall cue}

Sherin ${ }^{4}$ notes that recall is not a symbolic form because it is not necessarily connected with a conceptual understanding and does not have a single mathematical structure; nevertheless, it can be useful in problem solving. We found that students did use it to cue integration. We identify the recall cue in student work when a student simply states a fact such as "velocity is the derivative of position." This cue was used in the capacitor problem by five of the nine students. None of these students mentioned any notion of dependence or partsof-a-whole in the same problem. It should not be surprising that the capacitor problem was the only problem for which students used the recall cue because connecting velocity and acceleration through integrals and derivatives is a common application of calculus in physics classes and of physics in calculus classes.

Bruce integrated in the capacitor problem only after spending several minutes on another unproductive method. In the following quote we have italicized the phrases that indicate that he is using recall (with some difficulty) to cue integration: "Well, like, I was thinking, um...I was talking to [another student] and all those equations I didn't remember, well if you take the simplest one [acceleration] and keep integrating, you end up getting them all. But with this one...I was just thinking about it, but I'm not really sure how, how I would exactly start. Well I know, obviously, like, the derivative of position equals velocity, and the derivative of velocity equals, um...uh, hey. No, yeah, see, see...I'm getting, like, these equations popping up that don't actually come back. And then I'm just, like, nope they're not coming back. Like, I don't have a...so, and then, uh, velocity to acceleration...take the derivative...I don't know how to get from acceleration to force as far as calculus."

The other four students who used recall (Edward, Alex, Isaiah, and Esther) were able to recall more quickly and confidently. Edward's solution is typical of these responses: "if you use $F$ net equals $m a$...that will give us acceleration, which is the derivative of the velocity."

\section{Dependence cue}

Eight of the nine students used the symbolic form of dependence as a cue to use integration at least once. Sherin describes the dependence symbolic form as "a whole depends on the quantity associated with an individual symbol" and identifies it in student conversation as follows: "The observation that a particular symbol appears in the expression. Common phrases: depends on, is a function of. Also indicated by inferences, such as if $x$ varies, then the whole may vary." "We use Sherin's definition and identification scheme to define the dependence cue. Dependence is a piece of the conception of covariance that Thompson ${ }^{7}$ found to be essential in understanding functions and definite integrals.

In the nonconstant density problem, the seven students who integrated mentioned the dependence on $x$ as a cue to integrate. For instance, Ivan noted that "It looks like it's going to have to be an integral, because length is varying with the charge, [sic] so you were going to integrate the charge density...because the length is varying. So...you don't want to take it at every little point because there is an infinity amount of points between 0 and $0.5 \mathrm{~m}$." (Quote 1)

Ivan's explanation illustrates his thinking that length varies and because length can be measured at an infinite amount of points along the bar, an integral is needed to compute the total charge. Similarly, but more concisely, Edward, Alex, Georgette, Esther, and Isaiah described their use of dependence as a cue to integrate. Edward's explanation is typical of this way of thinking: "Um, so you would have to do an integral, cause you have to integral [sic] it cause well, cause it's changing with the distance $x$."

In the electric field problem, five students integrated and all used the dependence cue. Isaiah's explanation is typical of other students' responses: "I know that I am only going to do another integral, because the distance matters." We interpret "because distance matters" as Isaiah's way of saying that the density varies with the location on the bar.

In the capacitor problem two students were cued to integrate by dependence, but not in a way that we expected or that led to a solution. Both David and Georgette integrated force over time in an unsuccessful attempt to calculate the total force. In the following response from David, we see that he was trying to use integration to find a formula for the electric field, mistakenly beginning with the formula for the magnetic field. We have simplified this quotation, but kept intact all references to the integration process: "But [the distance] is changing. So you probably need to do an integral again. 'Cause that's going to be changing, it will be like 1.5, $1.3, \ldots$ 'Cause your, that's, all that's changing over time, so that makes it really complicated... That's how we do that. I guess you would want to take the integral because, again, you'd do like seven equations to plug in different [distances]. So we're doing the integral and saying, okay, it's from 0 , from 1.5 to 0 you get the entire force."

By integrating the total force over time, the result is change in momentum-the impulse-momentum theorem. However, there is no evidence that he is recalling the impulse-momentum theorem; rather he (re-)invented it in order to deal with a changing force. Georgette, who started to solve this problem in a manner similar to David, realized that integrating the force with respect to time would change the units, and she therefore abandoned this approach.

In summary, eight of the nine students used the dependence symbolic form in at least two problems to cue integration. Sherin's description of this symbolic form is very much in agreement with what we heard the students saying: Something changes (varies), and so integration must be used.

\section{Parts-of-a-whole cue}

Five of the nine students used the symbolic form of partsof-a-whole to cue integration at some point in solving the problems; in many instances it was in combination with dependence. Sherin describes parts-of-a-whole as "amounts of generic substance, associated with terms, that contribute to a whole" and identifies it "unlike competing terms, these entities are not influences. Utterances enumerate the parts that contribute to the whole, sometimes in correspondences with a diagram. Also indicated by inferences, such as the observation that if one part increases and the others are held fixed, then the whole increases." Interpreting the integral as a sum of many contributions, without any reference to balancing or canceling, requires invoking the parts-of-a-whole resource.

In the nonconstant density problem, four students, Alex, Esther, Georgette, and Isaiah, indicated that integration was required because they needed to add up charges. Alex explained the need to integrate as follows: "If you have just a little bit, just look at a little chunk of the bar and assume that 
the charge density over that little chunk is constant. Then you would call that little chunk the thickness of it, the length of it, $d x$. And so that is your, so the charge due to that little chunk is the charge density at the beginning times the thickness of the chunk so you just add them all up all the way along the bottom and use an integral to do that." (Quote 2)

Alex describes the object that is being added up as formed multiplicatively; that is, "the charge density at the beginning times the thickness of the chunk." Thus, not only does Alex recognize that he is accumulating objects, but he also identifies how the objects are formed (through multiplication). Thompson and Silverman ${ }^{22}$ point to such multiplicative reasoning as an important aspect of understanding the Riemann sum.

In the electric field problem, three students, Alex, Esther, and Kevin, used both parts-of-a-whole and dependence as cues to integrate. Alex noted, "So the $E$ field due to this chunk...is something and then you add that, the contribution due to each chunk...because the $r$ for each chunk is different." Alex mentions both parts-of-a-whole ("and then you add that, the contribution due to each chunk") and dependence ("the $r$ for each chunk is different"). Kevin initially used the center of charge method for this problem, but once he understood that the charge was evenly spread over the bar, he used integration. Kevin (along with all of the other students in the conventional physics course) did not have direct instruction on integration to calculate electric fields. Thus, he essentially reinvented this method of solution. He explained, "Then you'd have to integrate, you'd have to integrate over the distance from there to there...cause you have to take the sum of the electric fields from each part."

In solving the capacitor problem, only Kevin invoked parts-of-a-whole. He found that acceleration was necessary to solve the problem. Furthermore, he understood that he could not use standard kinematics equations because the acceleration was not constant. Kevin again appealed to partsof-a-whole resource when he proposed an alternative approach to solving the capacitor problem: "I guess you got to do the sum of all the accelerations" to get the velocity. $\mathrm{He}$ then changed his mind: "No, I think we use energy when it's a variable acceleration." We see evidence that Kevin was reflecting on past problem solving experiences when he stated, "for the most part I have always just used energy [when acceleration was not constant]."

\section{E. Misapplication of symbolic forms}

Sometimes students misapplied symbolic forms. For example, the notion of dependence as a cue to integrate can cause some problems. Edward, Kevin, and Isaiah wrote $k q d r / r^{2}$ in the integral instead of $k d q / r^{2}=k \lambda d r / r^{2}$, simply sticking in a $d r$ next to the formula for a point particle. When questioned, the students said that they chose to integrate with respect to $d r$ because $r$ was changing, not noticing that the units and physical meaning of the integrand had changed. The use of the dependence symbolic form in this problem appears to be a dead end because students cannot reason using this resource that they need to use $\lambda d x$ instead of $q d x$. Put a different way, this resource is appropriate only when the quantity that changes is a density or rate of change; otherwise (as in the case of the electric field) it will yield an incorrect result.

Similarly, the parts of a whole symbolic form is misused in the capacitor problem. Both Kevin and Esther talked about "adding up the acceleration" to get the velocity. This application of the symbolic form is not quite right: Adding up the changes in velocity (acceleration $\times d t$ ) gives the total change in velocity. Additionally, Georgette and David integrated the electric field to find the total force (they were using both dependence and parts-of-a-whole symbolic forms). Georgette correctly noted that integrating force with respect to time would yield an answer with units different from force (it is the total change in momentum). David did not know what to do with what he believed to be the total force because he had forgotten the role of acceleration.

\section{F. Larger study}

To check our conclusions based on this small group, we asked 144 students in a calculus-based physics course (who had just spent a few classes learning how to find the electric field due to several point charges) to state why integration would be necessary to find the electric field due to a bar of charge, reminding them that integration was not necessary for a point charge. This question differs from our original questions. It is a preinstruction question which indicates that integration is necessary and asks why this use is needed. The goal was to see what cued integration for these students.

The majority of responses reflected results from our interviews: 53 of the 144 students made use of the dependence symbolic form, seven students used the parts-of-a-whole symbolic form, 13 students felt that integration was necessary to find the centroid of the bar (the synecdoche resource), and 16 students noted that the field was due to an infinite number of points, or that the whole bar needed to be taken into consideration. This last response is similar to Ivan's justification for integrating when the charge density is not constant: "So...you don't want to take [the charge density] at every little point because there is an infinity amount of points between 0 and $0.5 \mathrm{~m}$." None of these 17 students (including Ivan) explicitly invoked parts-of-a-whole resource.

There were also some new responses. Thirty students believed that integration was necessary to find the area of the bar, and the area was in turn essential to find the field. Nine students felt that the "spreading out" of the charge required integration without elaborating further on the connection between this feature and the need for integration. The other 15 answers were each unique. We did not see the "area" response in our interviews, and speculate that this response was cued by a picture of the bar with a visible thickness (in our interviews the questions were not accompanied by pictures). We speculate that these students did not yet understand why integration was necessary, and made the one connection that was visibly obvious: The area of the bar. Spreading out was sometimes mentioned in connection with other ideas: Needing to take all the points into account or needing to calculate the area.

These data support our earlier results that the dependence symbolic form is the most common cue for integration, and the parts-of-a-whole cue is activated by fewer students. It also indicates that the synecdoche resource is persistent, even when students are told a problem requires integration.

\section{G. Section summary}

We have shown that students use symbolic forms to cue the use of integration in a physics context, and that these symbolic forms are used even in cases where recall was pos- 
sible (for example, for the impulse-momentum theorem). The most common resource used was dependence, followed by parts-of-a-whole. We presented evidence that symbolic forms can cue integration in a novel situation and allow students to invent equations.

Students typically do not pay attention to units when they choose the variable of integration; instead they pay attention to what is changing. We assert that this mistake is not trivial: It arises from students' strong reliance on the symbolic form of dependence as a cue for integration. This need to attend to units is similar to the multiplicative nature of the integrand, providing an additional challenge to students. ${ }^{22}$

Integration is most often used in calculus classes to find the area under a curve or to find the total amount of a quantity when its density or rate of change is nonconstant. In finding the electric field due to a bar of charge, neither of those meanings of integration is appropriate; instead integration must be viewed as adding up infinitesimal physical effects to obtain a total physical effect. This interpretation of the integral is a nontrivial change from the interpretations given in calculus class. There is another mismatch between mathematics and physics classes: In mathematics courses typically units are often not considered. However, in calculating the electric field, for example, it is essential to distinguish between charge and change density; one gives the correct units and the other does not. From these two important examples, we claim that physics is not a straightforward application of mathematics already learned; it is a reinterpretation of mathematical conventions in the context of physical principles. $^{23,24}$

\section{SPECULATION ON NESTING OF RESOURCES}

Based on our data analyses we conjecture that the dependence symbolic form is used by students developmentally before they use the parts-of-a-whole symbolic form. We base this conclusion on the number of students using each resource, the clarity of speech associated with each resource, and correlation between the parts-of-a-whole resource and the sophisticated notion of a limit.

We hypothesize that dependence is used before parts-of-awhole because three students (Ivan, David, and Edward) used dependence as a cue but did not talk productively about sums. For example, in Quote 1, Ivan did not explicitly discuss summing in his response to the nonconstant density problem. In contrast, all students who used parts-of-a-whole (Esther, Alex, Isaiah, Kevin, and Georgette) also used dependence as cues to integrate. We observed more clearly articulated responses from students who used the parts-of-a-whole symbolic form. For instance, Alex in Quote 2 clearly discussed summation in his response to the nonconstant density problem. We take this clarity of explanation as evidence that these students have more experience and facility with the ideas involved, indicating greater cognitive development on this topic.

Three students who used parts-of-a-whole (Esther, Isaiah, and Georgette) also mentioned limits either directly or indirectly in their explanations. For example, Esther notes: "The charge density describes the charge per tiny little pieces and since that is changing, I need to add up all the densities in all the little pieces, which is a Riemann sum, which as you let the width of the pieces go to zero, becomes the integral over the length of the bar." As discussed in Sec. II the concept of limit is difficult for many students. Therefore, we conclude that those students who invoked the idea of limit are students with stronger conceptual understanding.

We infer that two of the students who did not mention limits were struggling with the notion of limits in ways documented in Sec. II. For instance, David (who never talked about limits) explicitly talked about sums in his response to the nonconstant density problem, but noted that the sum (which he referred to as a set of equations) "won't even give you an exact amount."

We encourage future research efforts to further examine the developmental nesting of dependence, parts-of-a-whole, and other symbolic forms.

\section{IMPLICATIONS FOR INSTRUCTION}

We would like for students to be cued to integrate by the parts-of-a-whole symbolic form because this resource is more powerful and flexible in many physics contexts than the dependence symbolic form. Yet we have seen that partsof-a-whole is not a cue for most students with methods of direct instruction. ${ }^{17} \mathrm{We}$ designed instruction ${ }^{25}$ to promote this goal while taking into account common student difficulties and tested this activity on the same group of 144 students discussed in Sec. VI. The activity was done by groups of students during the regular 80-min weekly problem solving session.

Our first strategy was to use methods of guided discovery, promoting student reinvention of a formula for the electric field due to a bar of charge using the parts-of-a-whole resource. The first step was to invoke this resource in a simpler context: The students were asked to find the total charge on a bar with nonconstant density. Most integrated or found the area under the curve geometrically. Then they were presented with a quote from a fictitious student (using wording very similar to Alex's clear explanation in Quote 2) who noted that she solved the problem using the parts-of-a-whole symbolic form and were asked to evaluate the validity of her method. Forty-four out of 45 groups agreed that her method and reasoning were correct. The one group that disagreed had the same concern as Isaiah that the charge might not be evenly spread out over the bar. The result indicates that although most students do not spontaneously invoke the partsof-a-whole symbolic form, it is recognized as applicable when suggested. At the end of the tutorial, 30 groups were able to reinvent the correct expression for the electric field due to a bar of charge. Of the remaining groups, six groups integrated but used $q$ instead of $\lambda$, three groups took $r$ to be constant but integrated the constant charge density, and the remaining five groups had various difficulties.

To show the power of the parts-of-a-whole symbolic form, it would be useful to return to these ideas in several other contexts: The electric field due to a bar of nonconstant density, the electric potential due to a charge distribution, the electric field due to a bar of charge where vector issues are important, and the Biot-Savart law. In each successive application of the parts-of-a-whole resource, students should be given less and less guidance as they reinvent the appropriate integral.

Another strategy is to lead students to discover the inappropriateness of the synecdoche resource in this context. By comparing the value of the electric field using the center of charge method and the integral, students were prompted to note that the electric field using these methods is not the 
same. Students are asked if the center of charge method would work if the field's functional dependence on $r$ were proportional to $q r$ or $q / r$. At least a few students found this question natural and intriguing, and were surprised to discover that the synecdoche resource only applies for linear dependence. This (mis)application of the synecdoche resource is a case where the applicability of an intuitive resource must be verified quantitatively.

In addition to these strategies, instructors should be prepared to allow students to revisit the notion that the limit gives the exact sum; there will surely be a few students in each class for whom this idea is still a stumbling block. While not related to resources, we note that students often confused charge with charge density, and confuse $r$ (the distance between the charge and the field point) with $x$ (the location on the axis). Looking at cases where these quantities differ can help distinguish them.

\section{SUMMARY AND CONCLUSIONS}

The goal of our investigation was to explore the mathematical resources students invoke to guide their work as they integrate in the context of electrostatics. Based on our investigation, we have found evidence that students do invoke Sherin's symbolic forms in calculus contexts. In particular, students invoked the symbolic forms of dependence and parts-of-a-whole when they solved electrostatics problems that required integration, even in novel situations. Students are more likely to invoke the dependence symbolic form than parts-of-a-whole. However, the use of the dependence symbolic form led to inaccuracies if the quantity being integrated was not a rate or a density; in these cases, the units were always incorrect and few students noticed this error.

The parts-of-a-whole symbolic form is useful conceptually in physics when it is necessary to add little pieces of a physical quantity (for example, the electric field). This point of view is different from the standard presentation in a calculus class, which links integration to finding the area under a curve or finding the total amount given a rate or density. Therefore, students must reinterpret mathematics in a physics context, not simply apply known mathematical ideas.

Because the parts-of-a-whole symbolic form requires a reinterpretation of mathematics, students need to be guided to discover the parts-of-a-whole symbolic form as a useful way to solve problems requiring integration. They should also be guided to discover the applicability of parts-of-a-whole in different contexts to reinforce their understanding of this point of view.

Initial data indicate that students invoke the dependence symbolic form developmentally before parts-of-a-whole, possibly because of difficulty with the notion of limit. Research in mathematics education has shown that many students understand the limit only as an approximation to a sum and not as an accurate method to take an infinite number of values of the integrand into account. ${ }^{10}$ Therefore, it is not surprising that these ideas are challenging in a physics context as well. More research is needed to explore this conjecture.

Finally, we found that students' lack of understanding of physics concepts sometimes masks their mathematical understandings. For example, when students were confused about the location of the electrons, they often could not proceed with problem solving. Conversely, Aarons ${ }^{26}$ argued that a lack of mathematical proficiency may interfere with an understanding of physics concepts.

Our work opens more lines of investigation. Physics instructors often count on students to learn the requisite mathematics in their mathematics classes and expect them to effortlessly apply it in the physics context. But it is likely that such a transfer of skills does not happen as readily as we would hope because students must reinterpret mathematics in a physics context. What resources and skills do students bring from mathematics courses and what reinterpretation of the mathematics must be guided by their physics instructors? What resources are most productive in each application? Is this resource spontaneously invoked by students, or must we suggest its use to students?

For example, the Taylor series is taught in first year calculus; however, students do not readily understand when and why it is useful in a physics context. The use of the Taylor series requires scaled variables, which are typically not discussed in calculus and require explicit instruction in physics.

In such studies we must keep in mind that mathematical resources are not the only resources that affect student success in the physics classroom. Tuminaro et al.'s work gives a broad theoretical framework for understanding students' mathematical reasoning and understanding in the context of physics. ${ }^{27,28}$ In addition to mathematical resources (including Sherin's symbolic forms), they note that student expectations and patterns of activities are essential to understanding students' use of mathematics in a physics context. They conclude that there are many reasons for students' mathematical errors in physics in addition to students' lack of mathematical resources. Therefore, the correct diagnosis of student errors is necessary for effective teaching.

\section{ACKNOWLEDGMENTS}

The authors thank Christopher Bauer, Karen Graham, Christopher Shubert, and the two anonymous reviewers for careful readings and insightful suggestions that greatly improved this manuscript. This work was supported in part by NSF Grant No. DUE-9752485.

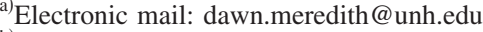

${ }^{b)}$ Electronic mail: karenmar@pdx.edu

${ }^{1}$ Andrea A. diSessa, "Toward an epistemology of physics," Cogn. Instruct. 10, 105-226 (1993).

${ }^{2}$ David Hammer, "Student resources for learning introductory physics," Am. J. Phys. 68, S52-S59 (2000).

${ }^{3}$ David Hammer, Andy Elby, Rachel Scherr, and Joe Redish, "Resources, Framing and Transfer," in Transfer of Learning from a Modern Multidisciplinary Perspective, edited by Jose Mestre (Information Age Publishing, Greenwich, CT, 2005).

${ }^{4}$ Bruce L. Sherin, "How students understand physics equations," Cogn. Instruct. 19, 479-541 (2001).

${ }^{5}$ Joan Ferrini-Mundy and Karen Graham, "An overview of the calculus curriculum reform effort: Issues for learning, teaching, and curriculum development," Am. Math. Monthly 98, 627-635 (1991).

${ }^{6}$ A. Orton, "Students' understanding of integration," Educ. Stud. Math. 14, 1-18 (1983).

${ }^{7}$ Patrick. W. Thompson, "Images of rate and operational understanding of the fundamental theorem of calculus," Educ. Stud. Math. 26, 229-274 (1994).

${ }^{8}$ S. Vinner, "The avoidance of visual considerations in calculus students," Focus on Learning Problems in Mathematics 11, 149-156 (1999).

${ }^{9}$ L. C. McDermott, "Oersted Medal Lecture 2001: Physics education research: The key to student learning," Am. J. Phys. 69, 1127-1137 (2001).

${ }^{10}$ B. Cornu, "Limits," in Advanced Mathematical Thinking, edited by
} 
David Tall (Kluwer Academic, Boston, 1991), pp. 153-166.

${ }^{11}$ Lillian C. McDermott and Edward F. Redish, "RL-PER1: Resource letter on physics education research," Am. J. Phys. 67, 755-772 (1999), and references therein.

${ }^{12}$ David Tall and Shlomo Vinner, "Concept image and concept definition in mathematics with particular reference to limits and continuity," Educ Stud. Math. 12, 151-169 (1981).

${ }^{13}$ Steven Williams, "Models of limits in college calculus students," J. Res. Math. Educ. 22, 219-236 (1991).

${ }^{14}$ Michael Oehrtmam, Ph.D. thesis, University of Texas, Austin, 2002.

${ }^{15}$ D. O. Tall and R. L. E. Schwarzenberger, "Conflicts in the learning of real numbers and limits," Math. Teach. 82, 44-49 (1978).

${ }^{16}$ Randall Harrington, Ph.D. thesis, University of Washington, 1995.

${ }^{17}$ Stephen Kanim, Ph.D. thesis, University of Washington, 1999.

${ }^{18}$ Michael C. Wittmann, "The object coordination class applied to wavepulses: Analysing student reasoning in wave physics," Int. J. Sci. Educ. 24, 97-118 (2002).

${ }^{19}$ K. A. Marrongelle, Kelly Black, and Dawn Meredith, "Studio Calculus and Physics: Interdisciplinary Mathematics with Active Learning," in Integrated Mathematics: Choices and Challenges, edited by Sue Ann McGraw (National Council of Teachers of Mathematics, Reston, VA 2003), pp. 103-115.

${ }^{20}$ David Halliday, Robert Resnick, and Jearl Walker, Fundamentals of Physics (Wiley, New York, 2003), 6th ed.; Raymond A. Serway and Robert J. Beichner, Physics for Scientists and Engineers (Saunders Col- lege Publishing, Fort Worth, 2000), 5th ed.

${ }^{21}$ There was a typographical error in this question. The electric field should have been $E(t)=8 \sin \left(5 \times 10^{6} t\right) \mathrm{N} / \mathrm{C}$. As it stands, the electric field is essentially 0 during the very short time inside the plates. Only Georgette realized this error and was somewhat confused by it.

${ }^{22}$ Patrick W. Thompson and Jason Silverman, "The Concept of Accumulation in Calculus," in Making the Connection: Research and Teaching in Undergraduate Mathematics, edited by M. Carlson and C. Rasmussen (Mathematical Association of America, Washington, DC) (in press).

${ }^{23}$ Corinne A. Manogue, Kerry Browne, Tevian Dray, and Barbara Edwards, "Why is Ampere's law so hard? A look at middle-division physics," Am. J. Phys. 74, 344-350 (2006).

${ }^{24}$ E. F. Redish, "Problem Solving and the Use of Math in Physics Courses," in Proceedings of the Conference on World View on Physics Education 2005: Focusing on Change, Delhi, India. (to be published), arXiv:physics/0608268.

${ }^{25}$ The tutorial and homework are available at $\langle$ pubpages.unh.edu/ $\sim$ dawnm/ connectm\&m.html $>$.

${ }^{26}$ Arnold B. Aarons, A Guide to Introductory Physics Teaching (Wiley, New York, 1990).

${ }^{27}$ Edward F. Redish, Rachel E. Scherr, and Jonathan Tuminaro, "Reverseengineering the solution of a simple physics problem: Why learning physics is harder than it looks," Phys. Teach. 44, 293-300 (2006).

${ }^{28}$ Jonathan Tuminaro, Ph.D. thesis, University of Maryland, College Park (2004).

\section{ONLINE COLOR FIGURES AND AUXILIARY MATERIAL}

AJP uses author-provided color figures for its online version (figures will still be black and white in the print version). Figure captions and references to the figures in the text must be appropriate for both color and black and white versions. There is no extra cost for online color figures.

In addition AJP utilizes the Electronic Physics Auxiliary Publication Service (EPAPS) maintained by the American Institute of Physics (AIP). This low-cost electronic depository contains material supplemental to papers published through AIP. Appropriate materials include digital multimedia (such as audio, movie, computer animations, 3D figures), computer program listings, additional figures, and large tables of data.

More information on both these options can be found at www.kzoo.edu/ajp/. 